Nicola Würffel (Leipzig)

\title{
Soziale Medien im
}

\section{Deutsch-als-Fremdsprache-Unterricht: Potenziale und Herausforderungen}

\begin{abstract}
Zum (Fremdsprachen-)Lernen mit Sozialen Medien existieren verschiedene Hypothesen, von denen ein paar eine große Stoßkraft entwickelt haben, obwohl sie sich empirisch nicht belegen lassen. In diesem Artikel werden einige von ihnen dekonstruiert, gleichzeitig wird aber auch gezeigt, warum und mit welchen Zielen ein Einsatz Sozialer Medien im Deutsch-als-Fremdsprache-Unterricht sinnvoll ist. Möglichkeiten des Einsatzes werden beispielhaft für die Förderbereiche des kooperativen Schreibens sowie für eine Verbindung von informellem und formalem Lernen thematisiert. Dabei wird ein besonderer Schwerpunkt auf die Betrachtung sozialer Netzwerke gelegt. Abschließend wird auf Herausforderungen eingegangen, die sich im Zusammenhang mit dem Einsatz Sozialer Medien im Deutsch-als-Fremdsprache-Unterricht stellen.
\end{abstract}

\section{Einleitung}

In bildungspolitischen und bildungswissenschaftlichen Diskursen über den Einsatz Sozialer Medien zum Lernen allgemein und zum Fremdsprachenlernen im Spezifischen wird gern von folgenden Hypothesen ausgegangen:

- Soziale Medien spielen im Leben der meisten Menschen in Deutschland und weltweit eine große Rolle.

- Weil Soziale Medien im privaten Leben von Lernenden eine so wichtige Rolle spielen, sollten sie auch in Lehr-Lernkontexten eingesetzt werden, um Lebenswelt und Lernwelt zu verbinden.

- Je jünger die Menschen sind, desto eher kann man davon ausgehen, dass sie „Digital Natives“ sind (bzw. der Net-Generation angehören) und damit als medienkompetent angesehen werden können.

- Weil soziale Netzwerke im Leben so vieler Menschen eine wichtige Rolle spielen, kann man zudem davon ausgehen, dass die Menschen inzwischen anders lernen: Wenn Kooperation mit anderen im Privaten wichtig ist, stehen Lernende kooperativen Arbeits- und Lernformen positiver gegenüber und können diese effizient einsetzen. 
Diese Hypothesen lassen sich unterschiedlich verifizieren bzw. falsifizieren. Nachdem in Kapitel 2 kurz erläutert wird, was in diesem Artikel unter Sozialen Medien und sozialen Netzwerken verstanden wird, werden in Kapitel 3 einige der oben genannten Hypothesen diskutiert, um auf diese Weise zu begründen, warum eine Beschäftigung mit Sozialen Medien für den Unterricht in Deutsch-als-Fremdsprache (DaF) dringend nötig erscheint. In Kapitel 4 wird thematisiert, mit welchen Zielen Soziale Medien im DaF-Unterricht eingesetzt werden können bzw. sollten, und in Kapitel 5 werden deren Potenziale am Beispiel zweier Förderschwerpunkte ausgeführt. Die mit einem Einsatz verbundenen Herausforderungen werden in Kapitel 6 benannt. Ein kurzes Fazit schließt den Artikel ab.

\title{
2 Soziale Medien und soziale Netzwerke
}

\author{
Unter Sozialen Medien verstehe ich nach Büffel/Pleil/Schmalz
}

Publikations- und Kommunikationsformen [...], die nicht nur als Instrumente für das individuelle und kollaborative Wissensmanagement eingesetzt werden können, sondern neben der reinen Informationsverknüpfung auch dabei helfen, eine soziale Beziehung zwischen ihren Nutzern zu etablieren (Büffel/Pleil/Schmalz 2007, S. 1).

Soziale Netzwerke (mit Text-, Bild-, Audio- und/oder Videoteilfunktion) sind dabei die Form Sozialer Medien, die vor allem kontaktunterstützend bzw. profilbildend wirken, wohingegen andere Soziale Medien wie Foren, Wikis, Blogs und Microblogging-Dienste, Podcasts, Foto-, Video- und Audiotauschplattformen etc. stärker zu den informationsorientierten Sozialen Medien gehören (vgl. u.a. Reinhardt 2019, S. 17).

Eine andere mögliche Unterscheidung bezieht sich auf die von den Sozialen Medien genutzten Kodierungsformen: So unterscheiden Lomicka/Lord (2016) bei sozialen Netzwerken zwischen denen, die vor allem den schriftlichen Austausch unterstützen (Facebook, LinkedIn, Twitter), denen, die den mündlichen Austausch unterstützen (PodOmatic, VoiceThread), und denen, die dem Austausch von Bildern dienen (Instagram, Pinterest, Snapchat). Da neuere Soziale Medien zunehmend komplexer werden und mehrere Anwendungen zusammenfassen, werden solche Abgrenzungen aber immer schwieriger.

Um die Bedeutung Sozialer Medien für Lernende verschiedener Altersklassen in Deutschland darzustellen, wird gern auf die Ergebnisse der KIM- und JIM-Studie (für die Gruppen der Kinder und Jugendlichen) sowie auf die ARD/ZDF-OnlineStudie (für die Gruppe der Erwachsenen) verwiesen. Diese belegen, dass Soziale Medien und soziale Netzwerke für alle Gruppen in Deutschland im Privaten einen 
wichtigen Platz einnehmen: Der Messenger-Dienst WhatsApp (zum Verschicken von Textnachrichten, aber auch von Fotos oder Videos) ist das am häufigsten genutzte Werkzeug; es folgen Anwendungen, mit denen Fotos und Videos geteilt werden können (Instagram und Snapchat). Facebook wird von Jugendlichen in Deutschland dagegen inzwischen etwas weniger genutzt (die größte Nutzergruppe sind hier - das zeigt die ARD/ZDF-Online-Studie - die 21-29-Jährigen, bei denen der Facebook-Gebrauch unverändert hoch ist, vgl. Frees/Koch 2018, S. 410).

Interessant sind auch die detaillierten Ergebnisse zur Anwendung der Sozialen Medien bei den Jugendlichen: Bei Snapchat z.B. besteht die häufigste Nutzung im Anschauen der Produkte anderer Nutzer und Nutzerinnen; das Erstellen eigener Produkte nimmt zumindest bei der Nutzung der Foto- und Video-Austauschanwendungen einen viel geringeren Raum ein. Dem Charakter der sozialen Netzwerke entsprechend werden zudem vor allem Produkte persönlich bekannter Personen angeschaut; das Anschauen der Produkte öffentlich bekannter Persönlichkeiten ist viel weniger wichtig (vgl. mpfs 2018, S. 42).

\section{Beliebte Hypothesen zum Einsatz Sozialer Medien für das Lernen}

Die seit Jahren steigende intensive Nutzung digitaler Medien vor allem durch jüngere Menschen führt dazu, dass der schon vor 17 Jahren von Prensky geprägte Begriff der „Digital Natives“ (ebd. 2001, S. 1) oder der etwas später aufgekommene Begriff der Net-Generation weiterhin ungebremst genutzt werden:

Diese Generation [die Generation App - d.h. Personen, die mit Smartphones aufgewachsen sind] wird von einem technisch geprägten Blick auf Leben und Wirklichkeit bestimmt. Das „App-Denken“ beginnt mit der Idee, dass Handlungen im Leben der Menschen wie Algorithmen funktionieren: Bestimmte Aktivitäten führen zu berechenbaren Ergebnissen. (Ascherl/ Ballis 2017, S. 6)

Einer ganzen Generation von Menschen wird damit zum einen die Kompetenz zugesprochen, souverän mit Medien umgehen zu können, weil sie damit aufgewachsen ist; zum anderen werden Unterschiede im Lernverhalten postuliert wie bei Ascherl/Ballis zum Beispiel ein Mangel an Kreativität. Obwohl Schulmeister schon 2012 in einem Artikel zur Net-Generation eine Dekonstruktion des Begriffs der Digital Natives vorgelegt und zudem nachgewiesen hat, dass generationsbezogene Zuschreibungen prinzipiell nicht funktionieren (Schulmeister 2012), halten sich die Begrifflichkeiten beständig. Schulmeister und Loviscach sprechen deshalb von einer Mythenbildung: 
Die Grenze zwischen populären Darstellungen und ernstzunehmenden Studien zur Digitalisierung verschwimmt in der öffentlichen Wahrnehmung. Viele „commonsensical“ Annahmen gründen auf der Vorstellung, dass die Medien selbst für die Wirkung auf die Lernenden verantwortlich seien: „Most people working in this area are driven by an underlying belief that digital technologies are in some way capable of improving education" (Selwyn 2011, S. 713). Aus unreflektierten Alltagserfahrungen werden Mythen, die unser Verständnis vom Lernen mit Medien beeinflussen. (Schulmeister/Loviscach 2017, S. 1)

Dass sich die Begriffe nicht nur im gesellschaftspolitischen Diskurs finden, sondern auch in fachdidaktisch-wissenschaftlichen Publikationen und Diskussionen immer noch - meist völlig unkommentiert - auftauchen (siehe das Zitat von Ascherl/Ballis 2017 oben oder u.a. Hsien-Chin 2016, S. 485), ist umso befremdlicher, als es inzwischen ausreichend Studien gibt, die zeigen, dass die meisten (jüngeren) Lernenden zwar medienaffin sind, aber nicht (umfassend) medienkompetent; sie nutzen Lehr- und Lernmedien zudem nicht als Selbstzweck, sondern verstehen diese eher (arbeitsökonomisch gedacht) als Instrument zur Zielerreichung. Digitale Medien werden von diesen Lernenden meist sehr pragmatisch genutzt, häufig vor allem in der Zeit vor den Prüfungen und meist im Selbststudium. Die private Mediennutzung wiederum übersetzt sich nicht zwangsläufig in die Nutzung der Medien für das Lernen; dort ist das Medienverhalten eher konservativ. Von den Institutionen und Lehrenden erwarten die Lernenden häufig nicht mehr Medieneinsatz, sondern einen (in ihrem Sinne) besseren. Dieser sollte aus ihrer Sicht vor allem darauf abzielen, heute gängige Prüfungsformate bestehen zu können (siehe u.a. Dehne/Lucke/Schiefner-Rohs 2017, S. 78; Hess 2006).

Auch dass man mit Sozialen bzw. digitalen Medien anders lerne, kann in dieser Pauschalität nur als Mythos bezeichnet werden. Studien zeigen bislang nur: Mit Medien lernt man nicht schlechter als ohne (Langner 2017, S. 142). Das eigentliche Grundproblem dieses Mythos ist aber noch ein anderes: ,Lernen mit Sozialen Medien 'ist keine Methode; Soziale Medien sind (nur) Lehr- und Lernmedien unter vielen anderen, mit denen wiederum unterschiedlichste methodische Ansätze in völlig unterschiedlichen Kontexten umgesetzt werden. Allgemeine Aussagen zum Lernen mit Sozialen Medien sind deshalb gar nicht möglich. Betrachtet man methodisierte Werkzeuge (also zum Beispiel eine bestimmte Sprachlernapp auf einem Smartphone), dann müssen feststellbare Effekte immer in Zusammenhang mit dem didaktischen Design insgesamt gesehen werden - und nicht (nur) mit dem Einsatz des Mediums an sich.

Ein dritter Mythos betrifft die Fähigkeit zum kooperativen Lernen, über die jüngere Lernende angeblich in hohem Maße verfügen sollen: Die DNA der Generation Y ist kollaborativer: Studierende lösen Aufgaben heute eher im Team als allein (Dräger/Friedrich/Müller-Eiselt 2014, S. 5). 
Auch wenn es erfreulich wäre, träfe das tatsächlich zu, zeigen Studien, dass die Übertragung aus dem privaten ins professionelle Handeln kein Automatismus ist und dass altersbezogene Zuschreibungen fehl am Platz sind. In Würffel (2011) habe ich die Ergebnisse einer Studie zur virtuellen kooperativen Zusammenarbeit von spanischen und deutschen DaF-Studierenden vorgestellt, die zeigen, dass Kooperations- und Gruppenprozesse im digitalen Raum Studierende vor große Herausforderungen stellen: Sie kämpfen mit der geringeren sozialen Präsenz der Gruppenmitglieder und dem Einfluss der Kanalreduktion der verwendeten Medien auf die Kommunikationsprozesse, die deshalb expliziter und/oder aufwendiger angeregt werden müssen. Insgesamt beschreiben sie die Gruppenabstimmungsprozesse beim Online-Arbeiten als zunächst ungewohnt, schwierig, chaotisch, aufwendig und anfällig für Missverständnisse - erst mit der Zeit schaffen es die Gruppen, konfliktfreier miteinander zu arbeiten. Als hinderliche Aspekte nennen die Studierenden zudem die geringere Verbindlichkeit beim Online-Arbeiten (wobei andere aber auch von einem stärkeren sozialen Druck durch die höhere Sichtbarkeit der Beiträge der einzelnen Gruppenmitglieder berichten) und den unterschiedlichen Arbeitsrhythmus der Gruppenmitglieder.

Von einer kollaborativen DNA kann also nicht die Rede sein: Lernende müssen - wie immer schon - an das kooperative Arbeiten herangeführt und darin trainiert werden. Wichtig erscheint dabei auch, dass der Gewinn für das Produzieren erhöht und eine kooperative Autorenschaft eingeübt sowie honoriert werden muss. Die Integration computergestützten kooperativen Arbeitens sollte zudem für längere Zeiträume geplant und vorgenommen werden (Würffel 2011).

Die hohe Beständigkeit dieser (und vergleichbarer) Mythen sowie die unzureichende Wahrnehmung vorhandener Forschungsergebnisse zum Mediennutzungsverhalten und den tatsächlichen Medienkompetenzen von Lernenden hat wiederum Auswirkungen auf die Unterrichtspraxis an Schulen und Hochschulen. Die verbreitete Annahme, dass Digital Natives mit fortgeschrittenen Medienkompetenzen in die Schulen und an die Hochschulen kämen und deshalb in diesen nicht mehr gefördert werden müssten, hat dazu geführt, dass die Vermittlung von Medienkompetenzen und die inhaltliche Beschäftigung mit der Rolle von digitalen Medien für die verschiedenen Bereiche des Fremdsprachenlernens bislang sowohl in DaF-Curricula als auch in der Ausbildung von DaF-Lehrenden unzureichend verankert sind. Das ändert sich gerade langsam, auch weil die Vermittlung einer fächerübergreifenden wie auch einer fachbezogenen digital literacy in bildungspolitischen Papieren weltweit immer stärker gefordert (für Deutschland siehe das entsprechende KMK Papier von 2016) und das Erstellen von diesbezüglichen Kompetenzrastern bildungspolitisch unterstützt wird (siehe u.a. 
Vuorikari et al. 2016 für den DigComp und Redecker/Punie 2017 für den DigCompEdu). Bei der Erstellung dieser Kompetenzraster muss auch bedacht werden, dass sich die notwendigen Kompetenzen und damit die Lernziele in Zeiten einer zunehmenden Digitalisierung der Gesellschaft schon verändert haben und noch stärker verändern müssen (Krommer 2018): Sie sollten grundlegend überdacht und auch an neue oder veränderte Textsorten, Kommunikationsformen und Sprachverwendungskontexte im Bereich Deutsch als Fremdsprache angepasst werden.

\section{Ziele für den Einsatz Sozialer Medien im DaF-Unterricht}

Digitale Medien wurden in der Fremdsprachendidaktik sehr früh im Sinne Sozialer Medien verstanden - schon zu einer Zeit, als es den Begriff der Sozialen Medien und die meisten der darunter gefassten Anwendungen noch gar nicht gab. So ging es z.B. in einem der ersten größeren Projekte zum Einsatz digitaler Medien im Fremdsprachenunterricht DaF um die Begegnung von Sprachlernanfängern und -anfängerinnen mit Zielsprachensprechenden bzw. -lernenden: Das Projekt „Das Bild der Anderen“ entstand 1989 im Rahmen des European Schools Project (ESP), das 1988 von Mitarbeitern und Mitarbeiterinnen der Universität Amsterdam gegründet worden war und bis heute existiert (http://www.espnet.eu/image/).

Schon früh also wurde in der Fremdsprachendidaktik der Wert der digitalen Medien nicht nur als Informationsmedien, sondern vor allem auch als Kommunikations-, Interaktions-, Produktions- und Publikationsmedien erkannt. Die Wahrnehmung der Breite der Anwendungsmöglichkeiten verstärkte sich noch einmal mit dem Aufkommen der Sozialen Medien zu Beginn der 2000er Jahre - dies zeigt sich auch in einem Anstieg der diesbezüglichen Forschungsliteratur ab 2008/9 (siehe u.a. Würffel 2008; Lomicka/Lord 2009; Thomas (Hg.) 2009). Vom Einsatz Sozialer Medien versprach und verspricht man sich eine Vereinfachung, Vervielfachung und vor allem auch Veränderung der Qualität der Möglichkeiten zur Interaktion und Produktion beim fremdsprachlichen Lernen. Zunehmend wird zudem in den Blick genommen, dass über den Einsatz bzw. die Nutzung von Sozialen Medien eine Verbindung zwischen formalem und informellem Lernen hergestellt werden kann. Eng damit verknüpft ist wiederum die Hoffnung, durch den Einsatz Sozialer Medien das selbstgesteuerte oder autonome Lernen der Lernenden zu fördern. Wie das im Einzelnen aussehen kann, wird im Folgenden anhand ausgewählter Kompetenz- und Interaktionsbereiche verdeutlicht. 


\section{Einsatz Sozialer Medien im DaF-Unterricht}

Anwendungen, die als Soziale Medien und/oder soziale Netzwerke bezeichnet werden, werden im Fremdsprachenunterricht inzwischen zu sehr unterschiedlichen Zwecken eingesetzt; die Darstellung möglicher Potenziale und Herausforderungen ihres Einsatzes für das DaF-Lernen kann deshalb nur ausschnitthaft erfolgen. Ausgewählt wurden die Förderung des Schreibens und die Verbindung von formalem und informellem Lernen, da damit zwei sehr unterschiedliche Aspekte des Fremdsprachenlehrens und -lernens abgedeckt werden.

\subsection{Förderung des (kooperativen, kreativen oder identitätsbildenden) Schreibens}

Im Zusammenhang mit dem Einsatz Sozialer Medien im DaF-Unterricht ist schon früh auf deren Potenzial für das kooperative Schreiben hingewiesen worden. Untersuchungen und didaktische Vorschläge gibt es inzwischen für den Einsatz von kooperativen Texteditoren wie Wikis, aber auch zu sozialen Netzwerken wie Facebook oder didaktischen Netzwerken wie der Pasch-Net-Community (siehe u.a. Peterwerth 2014). Ein anderer Schwerpunkt liegt in der Nutzung Sozialer Medien für das Anregen des kreativen Schreibens. Hier liegen u.a. Vorschläge für Microblogging-Dienste wie Twitter vor (Schmidt/Hagelmoser/Saage 2010). Schließlich gibt es mit Blick auf die Nutzung von Blogs Vorschläge zur Unterstützung eines selbstreflexiven und selbstdarstellenden Schreibens (u.a. Vurdien 2013). Im Rahmen dieses Artikels wird nur die Förderung des kooperativen Schreibens in den Blick genommen und gezeigt, wie Wikis dafür eingesetzt werden können.

Bei Wikis handelt es sich um öffentliche oder durch Passwort geschützte Webseiten, die von den Nutzenden nicht nur gelesen, sondern auch direkt bearbeitet werden können. Die bekannteste Anwendung der Wiki-Software ist sicherlich Wikipedia; aber auch Angebote wie das ZUM-Wiki der Zentrale für Unterrichtsmedien sind in lokalen Kontexten inzwischen fest verankert.

Gut ein halbes Jahrzehnt nach dem Aufkommen von Wikis waren die Erwartungen an diese Form kooperativer Texteditoren in Bezug auf ihren Einsatz im Fremdsprachenunterricht zur Unterstützung des kooperativen Schreibens sehr hoch:

One of the most powerful benefits of using wikis in language learning is as a collaborative tool. Potentially, everyone in a group could work at changing and editing a document and work towards a final version of a particular text. This can help develop bonds between members of the class and create a community spirit. The premise here is that the learners can learn from each other, and learn through their interactions with other group members. (Sharma/Barrett 2007, S. 123) 
Man erhoffte sich also, dass durch die spezifischen Eigenschaften von Wikis Prozesse des kooperativen Schreibens besonders gut unterstützt werden könnten. Zu diesen Prozessen zählt man, dass Lernende sich bei dem für sie sehr problemträchtigen, kognitiv anstrengenden und zeitaufwändigen Prozess des Schreibens unterstützen, z.B. indem die Lernenden von dem in der Gruppe vorhandenen orthografischen, grammatischen, textgrammatischen, lexikosemantischen, textsortenspezifischen und/oder kulturellen Wissen profitieren, das alle gleichberechtigt in den zu verfassenden Text einbringen können; aber auch, indem sie für den gesamten Text nicht mehr allein verantwortlich zeichnen müssen, sondern die Verantwortung für positive wie negative Ergebnisse mit den anderen Gruppenmitgliedern teilen können (Reduzierung der Gefahr des Gesichtsverlusts). Durch das gemeinsame Schreiben soll für die Lernenden zudem eine Notwendigkeit geschaffen werden, viele Handlungsschritte zu verbalisieren und sich dadurch z.B. die eingesetzten Strategien bewusst zu machen. Auch die Möglichkeit zu gegenseitigen Korrekturen am gemeinsamen Text wird als Möglichkeit der gegenseitigen Unterstützung gesehen (funktioniert aber nicht immer aufgrund der starken Verankerung der individuellen Autorenschaft bei vielen Lernenden, siehe für eine Übersicht Würffel 2008).

Wikis bieten ein leicht zu benutzendes Werkzeug, um den gemeinsamen Schreibprozess einfach zu realisieren. Dabei können die Schreibenden sowohl am selben Ort sein (was der Verbalisierung von Handlungsschritten zugutekäme) als auch zeit- und ortsversetzt arbeiten (was einem unterschiedlichen Arbeitstempo der einzelnen Lernenden entgegenkommt, aber auch das kooperative Schreiben von Gruppen an unterschiedlichen Orten erst ermöglicht). Der Einsatz von Wikis kann den gemeinsamen Schreibprozess zudem durch die Möglichkeit zur permanenten Speicherung unterstützen, die alle Vorgänge der in den Wikis ablaufenden Zusammenarbeit sichert und damit langfristig verfügbar macht (wie z.B. die Vorschläge von Mitlernenden zur Erweiterung oder Verbesserung bestimmter Textteile; Bloh 2002, S. 159), wodurch Lernende die Vorgänge reflektierter rezipieren können. Lehrenden wiederum wird dadurch die Möglichkeit geboten, auf Gruppenarbeitsprozesse zuzugreifen, zu denen sie sonst keinen Zugriff haben, und diese gezielt zu unterstützen. Ein weiterer großer Vorteil von Wikis ist, dass damit Texte leicht veröffentlicht werden können: Man erhofft sich von dieser Öffentlichkeit einen Anreiz für die Lernenden, Texte von höherer Qualität zu erstellen. Dass Wikis erfolgreich zur Unterstützung kooperativen Schreibens eingesetzt werden können, zeigen inzwischen zahlreiche Publikationen (für ein Projekt für den Primarbereich siehe z.B. Anskeit 2012). 


\subsection{Verbindung von formalem und informellem Lernen}

Im Bereich des Fremdsprachenlernens erfährt der Begriff des informellen Lernens seit dem Aufkommen mobiler und Sozialer Medien größere Beachtung. Bevor man über den positiven Einfluss des informellen Lernens auf das formale Lernen sprechen kann, muss man den Begriff des informellen Lernens deutlicher fassen. In vielen Publikationen wird dafür auf die Definition der Europäischen Kommission von 2001 rekurriert, die das informelle Lernen vor allem vom Subjekt her fasst und mit einem nicht-intentionalen Lernen in Verbindung bringt.

Informelles Lernen: Lernen, das im Alltag, am Arbeitsplatz, im Familienkreis oder in der Freizeit stattfindet. Es ist (in Bezug auf Lernziele, Lernzeit oder Lernförderung) nicht strukturiert und führt üblicherweise nicht zur Zertifizierung. Informelles Lernen kann zielgerichtet sein, ist jedoch in den meisten Fällen nicht intentional (oder ,inzidentell“/beiläufig). (Europäische Kommission 2001, S. 33)

Der in dieser Defintion hergestellte Bezug zwischen informellem und nicht-intentionalem Lernen erscheint allerdings problematisch, da nicht-intentionales Lernen genauso gut in formalen und non-formalen Kontexten stattfinden kann. Zudem entspricht eine solche Definition offensichtlich nicht dem Verständnis vieler Lernender, wie Bohlinger in einer Untersuchung feststellen konnte. In dieser wurden Personen nach ihren Formen informellen Lernens gefragt. Als mögliche Formen wurde angegeben:

- Selbstständiges Lernen anhand von Unterlagen in gedruckter Form (Fachbücher, Fachzeitschriften etc.);

- computergestütztes Lernen bzw. Weiterbildung sowie internetgestützes Lernen;

- Lernen mit Hilfe von Bildungssendungen in Rundfunk und Fernsehen oder offline am Computer;

- Besuche von Einrichtungen zur Vermittlung von Bildungsinhalten (Bibliotheken, Lernzentren etc.) (Bohlinger 2009, S. 175).

Besser beschreiben lassen sich diese Konkretisierungen informellen Lernens mit einer Definition, die den Kontext als Bezugspunkt für eine Unterscheidung von informellem und formalem Lernen nutzt. Mit einer solchen Definition arbeiten Sauro und Zourou, die mit Bezug auf die Nutzung digitaler Medien im Fremdsprachenunterricht von einem learning in the wild sprechen und dieses als ein ,informal language learning that takes place in digital spaces, communities, and networks that are independent of formal instructional contexts" (Sauro/Zourou 2017, S. 186) definieren. Learning in the wild findet nach ihrem Verständnis in Kontexten statt, die soziale Interaktion unterstützen und die weniger kontrollierbar und gesteuert sind als ein institutioneller Unterricht. Für das DaF/DaZ-Lernen bieten diese 
Kontexte ,interesting, and perhaps even compelling, opportunities for intercultural exchange, agentive action, and meaning making“ (Thorne 2010, S. 144).

Tatsächlich zeigen Untersuchungen zur Nutzung Sozialer Medien für das Fremdsprachenlernen, dass Lernende selbst ihr zielsprachiges Interagieren in sozialen Netzwerken als Möglichkeit zum informellen und unstrukturierten Lernen ansehen (Selwyn 2008). Das zielsprachige Interagieren dient häufig nicht primär dem Spracherwerb, sondern eher sozialen oder identitätsbildenden Zwecken (Alm 2018). Lernende schätzen bei der Nutzung Sozialer Medien zudem die Möglichkeiten zur leichteren Vernetzung mit Zielsprachensprechenden und zum multimodalen Interagieren. Insgesamt werden Zugewinne vor allem im Bereich der interkulturellen und soziopragmatischen Kompetenzen gesehen sowie in der Möglichkeit zum zielgruppenspezifischen sprachlichen Produzieren (Reinhardt 2019).

Angesichts dieser Ergebnisse überrascht es wenig, dass sich die Fremdsprachendidaktik darüber Gedanken macht, wie man diese Kontexte und das darin stattfindende informelle Lernen auch für ein formales Lernen im gesteuerten Unterricht nutzbar machen kann. Erste Ergebnisse gibt es inzwischen u.a. zur Nutzbarmachung des Lernens mit/in Computerspielen (Sundqvist 2019), des Lernens in FanCommunities (Shafirova/Cassany 2019), von sozialen Netzwerken zur Förderung sozialer (fremdsprachlicher) Kontakte (Alm 2018) und von Facebook-Gruppen zur Förderung soziopragmatischer Diskurskompetenz (Sablotny 2017; Reinhardt 2019).

\section{Herausforderungen beim Lernen mit Sozialen Medien im Bereich DaF/DaZ}

Selbstverständlich ist ein Einsatz Sozialer Medien im DaF/DaZ-Unterricht auch mit vielen Herausforderungen verbunden. Auf drei Aspekte wird beispielhaft eingegangen: Dazu gehören die Nutzungsgewohnheiten der Lernenden, die starken Einfluss auf das (mögliche) Lernen mit den Medien nehmen, der Faktor Öffentlichkeit, mit dem man allzu schnell die Hoffnung auf eine Motivierung der Lernenden verbindet, und schließlich auf die Anerkennung von DaF-bezogenen Leistungen aus informellen Kontexten in formalen Kontexten, da dies noch ein weitgehend unbearbeitetes Feld darstellt (für weitere Herausforderungen vgl. u.a. Würffel 2008).

Wie schon oben ausgeführt, entspricht das Mediennutzungsverhalten von Lernenden in institutionellen Kontexten keineswegs ihrem Nutzungsverhalten außerhalb dieser Kontexte. Das liegt auch daran, dass der formale Charakter von Lernsituationen das Nutzungsverhalten im institutionellen Lernen maßgeblich beeinflusst (Lin/Groom/Lin 2013). Es ist zudem keineswegs sicher, dass Lernende in formalen Kontexten die motivierenden und eigentlich als lernförderlich wahr- 
genommenen Möglichkeiten der Sozialen Medien zu Lernzwecken überhaupt nutzen (wollen) (Waragai/Ohta/Raindl 2017). Einige Soziale Medien, die im Unterricht eingesetzt werden, werden wiederum privat gar nicht genutzt bzw. sind nicht bekannt (z.B. Wikis, siehe Rott/Weber 2013). Ein didaktisch sinnvoller Gebrauch Sozialer Medien muss deshalb auf jeden Fall immer explizit trainiert werden.

In Kapitel 4 wurde darauf hingewiesen, dass man sich beim Einsatz Sozialer Medien vom Faktor Öffentlichkeit sowohl eine Steigerung der Motivation der Lernenden als auch das Entstehen eines gewissen sozialen Drucks auf die Lernenden erhofft; beides soll dazu führen, dass eine bessere Qualität der Produkte erreicht werden kann. Vor allem in Bezug auf Wikis, aber auch mit Blick auf andere Soziale Medien, haben zahlreiche Studien inzwischen belegt, dass man gerade mit diesem Faktor vorsichtig und didaktisch reflektiert umgehen muss. Zwar ließ sich für einige Lehr-/Lernkontexte eine motivations- und/oder qualitätssteigernde Wirkung nachweisen, gleichzeitig wirkte die öffentliche Zugänglichkeit der Lernereinträge aber für einige Lernende auch einschüchternd sowie hemmend und führte dazu, dass sich diese Lernenden nur in geringem Umfang äußerten (Vurdien 2013). Mehrere Autoren und Autorinnen weisen deshalb darauf hin, dass Lernende an der Entscheidung beteiligt werden sollten, ob das fremdsprachliche, durch Aufgaben gesteuerte Schreiben in einem öffentlichen oder in einem passwortgeschützten Raum stattfinden soll (Guth 2007; Würffel 2008). Zudem sollte darauf geachtet werden, dass unterschiedliche Partizipationsgrade der Lernenden vorgesehen und erlaubt sind (Bradley/Lindström/Rystedt 2010).

Insbesondere im Bereich des Arbeitens mit Sozialen Medien erscheint es für den DaF-Unterricht sinnvoll, die in informellen Lernkontexten erfolgenden Lernprozesse für formale Kontexte stärker nutzbar zu machen. Dabei gibt es aber ein großes Hindernis:

Unfortunately, the considerable audience and register awareness, interactional, and identity performance skills involved in SNS use are usually neither recognized nor legitimized in traditional writing or language instruction. (Reinhardt 2019, S. 21)

Die Tatsache, dass informelle Lernergebnisse in formalen Lernkontexten nicht anerkannt werden und damit ihre Integration in diese erschwert wird, hat viel mit dem kulturbezogenen didaktischen Design dieser formalen Unterrichtskontexte zu tun. Anerkennungsmaßnahmen müssten deshalb systematisch integriert werden (die Änderungen also systemisch erfolgen) und nicht nur in Bezug auf einzelne digitale Medien. Ähnliches gilt auch für die Schaffung von Anreizen dafür, dass Lernende bei der Nutzung Sozialer Medien im DaF-Unterricht in deutlich stärkerem Maße zu Produzierenden werden, als sie das bei ihrem außerunterrichtlichen Umgang mit diesen Medien tun. 


\section{Fazit}

In einem konsequent handlungsorientierten und autonomiefördernden DaF-Unterricht könnten Soziale Medien lernunterstützend wirken. Ein solcher Unterricht (der zudem von Lehrenden und Lernenden gleichermaßen getragen wird) stellt aber nicht die (Unterrichts-)Realität an vielen Stellen der DaF-Welt dar. Ein gewinnbringender Einsatz Sozialer Medien im DaF-Unterricht kann deshalb erst dann stattfinden, wenn sich der ,formale“ DaF-Unterricht ändert - dafür müssten sich vielfältige Routinen aller Akteure und einige Rahmenbedingungen formaler Lernkontexte ändern. Allein schon die Bemühungen um Standardisierung und um eine kleinschrittige Überprüfbarkeit von Kompetenzen läuft dem entgegen. Das sollte man sich bewusstmachen, ehe man anfängt, nur über die Integration einzelner Sozialer Medien in den DaF-Unterricht nachzudenken, ohne dem ganzen Tableau aktueller DaF-Unterrichtsrealitäten genauere Aufmerksamkeit zu schenken.

\section{Literatur}

Alm, Antonie (2018): Advanced language learners as autonomous language users on Facebook. In: Buendgens-Kosten, Judith/Elsner, Daniela (Hg.): Multilingual computer assisted language learning. Bristol, S. 191-209.

Anskeit, Nadine (2012): WikiWiki in die Schule. Unterrichtsbeispiele und Praxiserfahrungen zum Einsatz von Wikis in der Schule. In: Beißwenger, Michael/Anskeit, Nadine/Storrer, Angelika (Hg.): Wikis in Schule und Hochschule. Boizenburg, S. 13-46.

Ascherl, Carina/Ballis, Anja (2017): Lernen mit Digitalen Medien. Chancen und Herausforderungen für schulisches Lernen. In: DaZ Sekundarstufe 2, S. 5-8.

Bloh, Egon (2002): Computerunterstützes kooperatives Lernen (CSCL). In: Lehmann, Burkhard/ Bloh, Egon (Hg.): Online-Pädagogik. (= Grundlagen der Berufs- und Erwachsenenbildung 29). Hohengehren, S. 146-182.

Bohlinger, Sandra (2009): Bildungspolitische Implikationen informellen Lernens. In: Bildungsforschung 6, 1, S. 159-184. Internet: https://core.ac.uk/download/pdf/33981309.pdf (Stand: 9.10.2019).

Bradley, Linda/Lindström, Berner/Rystedt, Hans (2010): Rationalities of collaboration for language learning in a wiki. In: ReCALL 22, 2, S. 247-265. Internet: https://core.ac.uk/ download/pdf/70583391.pdf (Stand: 9.10.2019).

Büffel, Steffen/Pleil, Thomas/Schmalz, Sebastian (2007): Net-Wiki, PR-Wiki, KoWiki Erfahrungen mit kollaborativer Wissensproduktion in Forschung und Lehre. In: kommunikation@gesellschaft 8, 2, S.1-23. Internet: https://nbn-resolving.org/ urn:nbn:de:0228-200708111 (Stand: 9.10.2019).

Dehne, Julian/Lucke, Ulrike/Schiefner-Rohs, Mandy (2017): Digitale Medien und forschungsorientiertes Lehren und Lernen - empirische Einblicke in Projekte und Lehrkonzepte. In: Igel, Christoph (Hg.): Bildungsräume. Proceedings der 25. Jahrestagung der Gesellschaft für 
Medien in der Wissenschaft. 5. bis 8. September 2018 in Chemnitz. (= Medien in der Wissenschaft 72). Münster, S. 71-83.

Dräger, Jörg/Friedrich, Julius-David/Müller-Eiselt, Ralph (2014): Digital wird normal. Wie die Digitalisierung die Hochschulbildung verändert. Gütersloh, S. 1-11. Internet: http://www. che.de/downloads/Im_Blickpunkt_Digital_wird_normal.pdf (Stand: 9.10.2019).

Europäische Kommission (2001): Ein europäischer Raum des lebenslangen Lernens. Luxemburg. Internet: https://eur-lex.europa.eu/LexUriServ/LexUriServ.do?uri=COM:2001:0678:FIN: DE:PDF (Stand: 9.10.2019).

Farr, Fiona/Murray, Liam (Hg) (2016): The Routledge handbook of language learning and technology. London/New York.

Frees, Beate/Koch, Wolfgang (2018): ARD/ZDF-Onlinestudie 2018: Zuwachs bei medialer Internetnutzung und Kommunikation. Ergebnisse aus der Studienreihe „Medien und ihr Politikum“ (MiP). In: Media Perspektiven 9, S. 398-413. Internet: www.ard-zdf-onlinestudie. de/files/2018/0918_Frees_Koch.pdf (Stand: 9.10.2019).

Guth, Sarah (2007): Wikis in education: Is public better? In: WikiSym '07: Proceedings of the 2007 International Symposium of Wikis, S. 61-68. Internet: http://equivocos.com/ wp-content/uploads/2014/02/Guth_WikiSym2007_IsPublicBetter.pdf (Stand: 9.10.2019).

Harsch, Claudia/Krings, Hans P./Kühn, Bärbel (Hg.) (2017): Inhalt und Vielfalt - neue Herausforderungen für das Sprachenlernen und -lehren an Hochschulen. Erträge des 5. Bremer Symposions. (= Fremdsprachen in Lehre und Forschung 50). Bochum.

Hess, Hans Werner (2006): „E-Lernen“ - Fakten und Fiktionen. In: Informationen Deutsch als Fremdsprache (Info DaF) 33, 4, S. 305-328. Internet: www.degruyter.com/view/j/ infodaf.2006.33.issue-4/issue-files/infodaf.2006.33.issue-4.xml (Stand: 9.10.2019).

Hsien-Chin, Liou (2016): CALL tools for reading and writing. In: Farr/Murray (Hg), S. 478-490.

Krommer, Axel (2018): Warum der Grundsatz „Pädagogik vor Technik“ bestenfalls trivial ist. In: Magazin Sprache des Goethe-Instituts. Internet: https://www.goethe.de/de/spr/mag/ 21451837.html (Stand: 9.10.2019).

KMK Papier (2016) = Kultusministerkonferenz: Bildung in der digitalen Welt. Strategie der Kultusministerkonferenz. Internet: https://www.kmk.org/fileadmin/Dateien/ veroeffentlichungen_beschluesse/2018/Strategie_Bildung_in_der_digitalen_Welt_idF._ vom_07.12.2017.pdf (Stand: 09.10.2019).

Langner, Michael (2017): (Sprachen-)Lernen trotz Medien-Hype! Anspruch und Wirklichkeit. In: Harsch/Krings/Kühn (Hg.), S. 141-148.

Lin, Ming Huei/Groom, Nicholas/Lin, Chin-Ying (2013). Blog-assisted learning in the ESL writing classroom: A phenomenological analysis. In: Journal of Educational Technology and Society 16, 3, S. 130-139.

Lomicka, Lara/Lord, Gillian (2009): The next generation: Social networking and online collaboration in foreign language learning. San Marcos, TX.

Lomicka, Lara/Lord, Gillian (2016): Social networking and language learning. In: Farr/Murray $(\mathrm{Hg})$, S. 255-267.

mpfs (2018) = Medienpädagogischer Forschungsverband Südwest: JIM-Studie 2018: Jugend, Information, Medien. Basisuntersuchung zum Medienumgang 12- bis 19-Jähriger. Internet: www.mpfs.de/fileadmin/files/Studien/JIM/2018/Studie/JIM_2018_Gesamt.pdf (Stand: 9.10.2019).

Peterwerth, Anna (2014): Fremdsprachenlernen in sozialen Netzwerken: Die SchülerCommunity der Website www.pasch-net.de. In: Fremdsprache Deutsch 51, S. 49-54. 
Prensky, Marc (2001): Digital natives, digital immigrants part 1. In: On the Horizon 9, 5. Internet: http://www.marcprensky.com/writing/Prensky\%20-\%20Digital\%20Natives,\%20 Digital\%20Immigrants\%20-\%20Part1.pdf (Stand 09.10.2019)

Redecker, Christine/Punie, Yves (2017): European framework for the digital competence of educators: DigCompEdu. Luxemburg. Internet: publications.europa.eu/en/publicationdetail/-/publication/fcc33b68-d581-11e7-a5b9-01aa75ed71a1/language-en (Stand: 9.10.2019).

Reinhardt, Jonathon (2019): Social media in second and foreign language teaching and learning: Blogs, wikis, and social networking. In: Language Teaching 52, 1, S. 1-39. Internet: doi.org/10.1017/S0261444818000356 (Stand: 9.10.2019).

Rott, Susanne/Weber, Elizabeth Dolly (2013): Preparing students to use Wiki software as a collaborative learning tool. In CALICO Journal 30, 2, S. 179-203. Internet: doi.org/ 10.11139/cj.30.2.179-203 (Stand: 9.10.2019).

Sablotny, Manfred (2017): „Danke für eure Tipps!“: Lernen auf der Basis sozialer Interaktion im DaF-Unterricht mit Facebook. In: Information Deutsch als Fremdsprache (Info DaF) 44, 1, S. 51-84.

Sauro, Shannon/Zourou, Katerina (2017): Call for Papers for CALL in the Digital Wilds Special Issue. In: Language Learning \& Technology 21, 1, S. 186. Internet: doi.org/10125/44666 (Stand: 9.10.2019).

Schmidt, Torben/Hagelmoser, Rebecca/Saage, Sven (2010): To tweet or not to tweet? Das Kommunikationstool Twitter im Fremdsprachenunterricht. In: Praxis Fremdsprachenunterricht 7, 4, S. 7-11.

Schulmeister, Rolf (2012): Vom Mythos der Digital Natives und der Net Generation. In: Berufsbildung in Wissenschaft und Praxis 41, 3, S. 42-46. Internet: www.bibb.de/ veroeffentlichungen/de/bwp/show/6871 (Stand: 9.10.2019).

Schulmeister, Rolf/Loviscach, Jörn (2017): Mythen der Digitalisierung mit Blick auf Studium und Lernen. In: Leineweber, Christian/de Witt, Claudia (Hg.): Digitale Transformation im Diskurs: Kritische Perspektiven auf Entwicklungen und Tendenzen im Zeitalter des Digitalen. Hagen, S. 1-21. Internet: ub-deposit.fernuni-hagen.de/servlets/MCRFileNodeServlet/mir_ derivate_00001263/DTiD_Schulmeister_Loviscach_Mythen_Digitalisierung_Studium_2017. pdf (Stand: 9.10.2019).

Selwyn, Neil (2011): Education and technology. Key issues and debates. London.

Selwyn, Neil (2008): An investigation of differences in undergraduates' academic use of the internet. In: Active Learning in Higher Education 9, 1, S. 11-22. Internet: doi.org/10.1177/ 1469787407086744 (Stand: 9.10.2019).

Shafirova, Liudmila/Cassany, Daniel (2019): Bronies learning English in the digital wild. In: Language Learning \& Technology 23, 1, S. 127-144. Internet: doi.org/10125/44676 (Stand: 9.10.2019).

Sharma, Pete/Barrett, Barney (2007): Blended learning. Using technology in and beyond the language classroom. Oxford.

Sundqvist, Pia (2019): Commercial-off-the-shelf games in the digital wild and L2 learner vocabulary. In: Language Learning \& Technology 23, 1, S. 87-113. Internet: doi.org/ 10125/44674 (Stand: 9.10.2019).

Thomas, Michael (Hg.) (2009): Handbook of research on Web 2.0 and second language learning, Hershey, PA u.a.

Thorne, Steven L. (2010): The 'Intercultural Turn' and language learning in the crucible of new media. In: Guth, Sarah/Helm, Francesca (Hg.): Telecollaboration 2.0. Language, Literacies 
and Intercultural Learning in the 21st century. (= Telecollaborations in Education 1). Bern, S. 139-164.

Vurdien, Ruby (2013): Enhancing writing skills through blogging in an advanced English as a foreign language class in Spain. In: Computer Assisted Language Learning 26, 2, S. 126-143. Internet: https://www.researchgate.net/publication/254216916_Enhancing_writing_skills_ through_blogging_in_an_advanced_English_as_a_Foreign_Language_class_in_Spain (Stand 09.10.2019).

Vuorikari, Riina/Punie, Yves/Carretero, Stephanie/Van den Brande, Lieve (2016): DigComp 2.0. The digital competence framework for citizens. Luxemburg. Internet: publications.europa. eu/en/publication-detail/-/publication/bc52328b-294e-11e6-b616-01aa75ed71a1/ language-en (Stand: 9.10.2019).

Waragai, Ikumi/Ohta, Tatsuya/Raindl, Marco (2017): Social Networking Services als Lernraum: Ein kontextsensitiver Text-Editor als Interface zwischen formalem und informellem Lernen. In: Harsch/Krings/Kühn (Hg.), S. 163-176.

Würffel, Nicola (2008): Kooperatives Schreiben im Fremdsprachenunterricht: Potentiale der Nutzung von Social-Software-Anwendungen am Beispiel kooperativer Online-Editoren. In: Zeitschrift für Interkulturellen Fremdsprachenunterricht 13, 1. Internet: tujournals.ulb. tu-darmstadt.de/index.php/zif/article/view/226/218 (Stand: 9.10.2019).

Würffel, Nicola (2011): Blended Learning als Lern- und Lehrform an deutschen Hochschulen. Ergebnisse einer Implementierung von Schule im Wandel in der Ausbildung angehender DaF-Lehrender. In: Würffel, Nicola/Padrós, Alicia (Hg.): Fremdsprachenlehrende aus- und fortbilden im Blended-Learning-Modus. Erfahrungen und Erkenntnisse aus dem ComeniusProjekt „Schule im Wandel“. Tübingen, S. 132-156. 